\title{
Electron Hologram Denoising via Sparse Coding and Dictionary Learning
}

\author{
Satoshi Anada $^{1 *}$, Yuki Nomura ${ }^{2,3}$, Tsukasa Hirayama ${ }^{1}$ and Kazuo Yamamoto ${ }^{1}$ \\ 1. Nanostructures Research Laboratory, Japan Fine Ceramics Center, Nagoya, Aichi, Japan. \\ 2. Technology Innovation Division, Panasonic Corporation, Moriguchi, Osaka, Japan. \\ 3. Department of Crystalline Materials Science, Graduate School of Engineering, Nagoya University, \\ Nagoya, Aichi, Japan. \\ * Corresponding author: s_anada@jfcc.or.jp
}

Electron holography is a phase imaging technique using a transmission electron microscope to quantitatively measure electric and magnetic fields at the nanometer scale. Electric and magnetic fields are recorded in an electron interference pattern (hologram), formed by overlapping the electron waves modulated by a specimen with those passing through a vacuum. The phase distribution of the modulated waves, which displays the electric potential and/or magnetic flux, can be reconstructed from the hologram. Since the noise in the hologram can deteriorate the precision of the phase measurement, denoising of the hologram is required to clearly visualize small field distributions. In the present study, to effectively reduce the noise in the hologram, we employed sparse coding and dictionary learning; we applied them to simple holograms of a GaAs p-n junction specimen recorded with different exposure times.

We performed sparse coding and dictionary learning using the scikit-learn Python package [1] for denoising holograms of a GaAs p-n junction specimen. The specimen was prepared by thinning one part of the bulk specimen using a cryo-focused-ion beam system (Hitachi NB5000). The holograms were acquired using an electron-holography TEM (Hitachi HF-3300EH) operated at $300 \mathrm{kV}$ with a multiple biprism system. We adjusted the TEM lens condition to form a fringe spacing of $12 \mathrm{~nm}$ and an interference region of $2 \mu \mathrm{m}$ in the specimen plane.

Figure 1 shows typical results of image denoising for holograms of GaAs p-n junction specimen with different exposure times; Figs. 1(a) and (b) are high-dose and low-dose original holograms, respectively, and Figs. 1(a') and (b') are the corresponding denoised holograms of Figs. 1(a) and (b), respectively. As clearly shown, the sparse coding and dictionary learning algorisms significantly reduce the noise in both of the holograms (Figs. 1(a) and (b)). The denoising process for high-dose hologram (Fig. 1(a)) indicates no significant change in the interference fringe patterns and enhances the clarity of the fringe bending at the p-n junction. Even though the noise level is much high in the low-dose original hologram (Fig. 1(b)), the fringe bending at the p-n junction is shown clearly in the denoised hologram (Fig. 1(b')).

To assess the denoising effect of the different dose holograms, we reconstructed the phase images from the original and denoised holograms. The reconstructed phase images are shown in Fig. 2; Figs. 2(a), (a'), (b) and (b') were reconstructed from Figs. 1(a), (a'), (b) and (b'), respectively. The phase difference between the $\mathrm{p}$ and $\mathrm{n}$ regions was observed in all of the phase images, even in the phase image (Fig. 2(b)), with a lot of noise, reconstructed from the low-dose original hologram (Fig. 1(b)). The phase images (Figs. 2(a') and (b')) reconstructed from the denoised holograms (Figs. 1(a') and (b')) exhibit no significant noise.

We extracted the phase profiles across the p-n junction from the regions indicated by the arrows in the 
phase images (Fig. 2), as shown in Fig. 3; Figs. 3(a) and (b) show the phase profiles extracted from the phase images (Figs. 2(a) and (a')) and (Figs. 2(b) and (b')), respectively. As shown in Fig. 3(a), the phase profile for the high-dose denoised hologram (Fig. 1(a')) includes no significant noise and is in excellent agreement with that for the corresponding original hologram (Fig. 1(a)). Furthermore, as shown in Fig. 3 (b), although the profile for the low-dose original hologram is too noisy to measure the phase shift of the p-n junction accurately, the profile for the denoised hologram includes less noise and is in better agreement with that for the high-dose holograms (Fig. 3(a)). These results indicate that the sparse coding and dictionary learning algorithms are effective for electron holography and can potentially improve the phase measurement precision and the temporal resolution [2].

References:

[1] F Pedregosa et al., J. Mach. Learn. Res. 12 (2011), p. 2825.

[2] The authors acknowledge funding from the Japan Society for the Promotion of Science (JSPS), Grant-in-Aid for Scientific Research KAKENHI [grant numbers JP 18K13795].
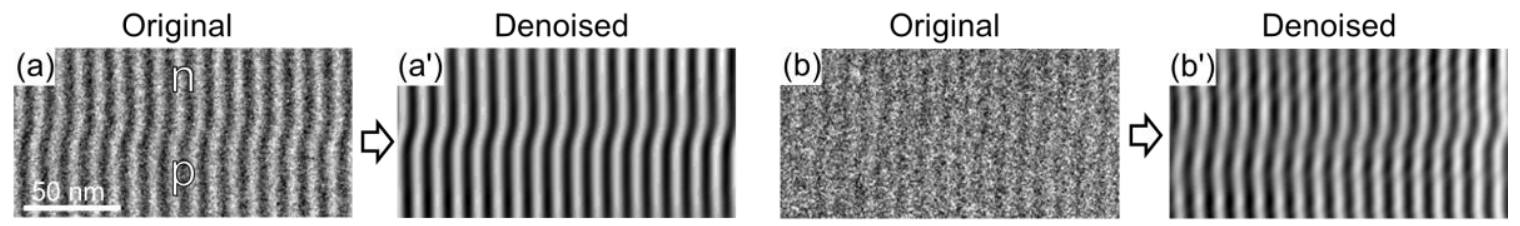

Figure 1. Hologram denoising for the GaAs p-n junction specimen. (a) and (b) are high-dose and original low-dose hologram, respectively. (a') and ( $\left.b^{\prime}\right)$ are the corresponding denoised holograms of (a) and (b), respectively.
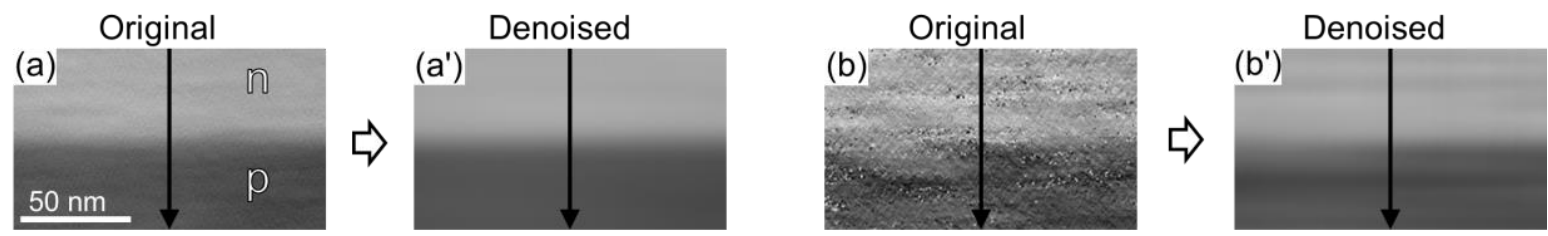

Figure 2. Phase images reconstructed from the original and denoised holograms (Fig. 1). (a), (a'), (b) and $\left(b^{\prime}\right)$ were reconstructed from Figs. 1(a), (a'), (b) and (b'), respectively.
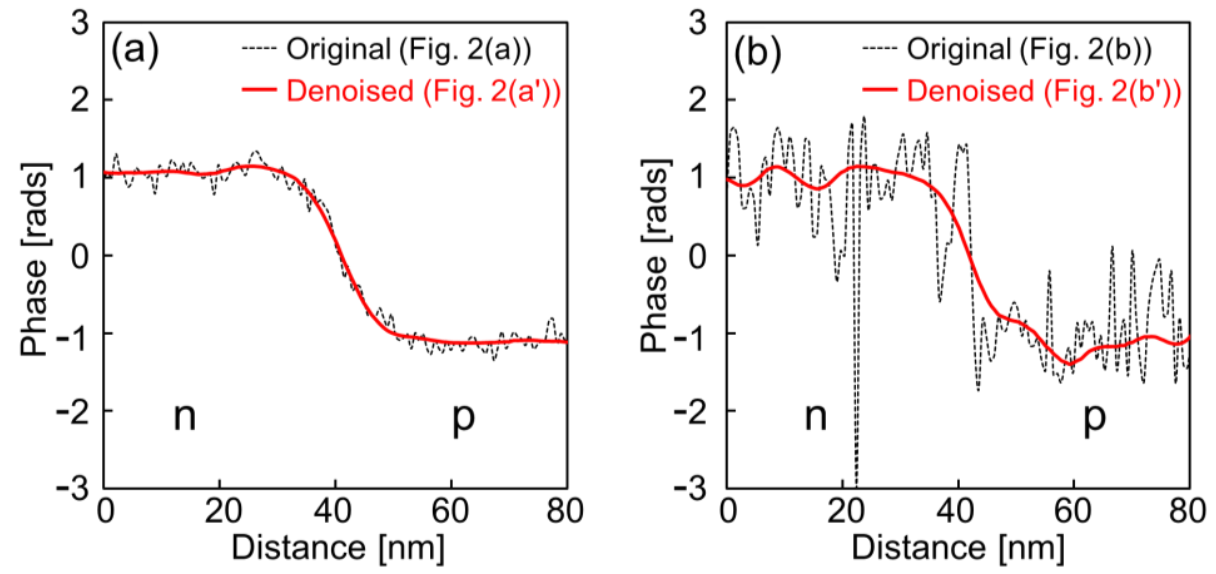

Figure 3. Phase profiles across the $\mathrm{p}-\mathrm{n}$ junction extracted from the regions indicated by the arrows in the phase images (Fig. 2); (a) and (b) show the phase profiles from the phase images (Figs. 2(a) and (a')) and (Figs. 2(b) and (b')), respectively. 